\title{
Tarif médical ambulatoire: la décision vous revient
}

\section{Christian Oeschger}

Collaborateur spécialisé, division Tarifs et conventions pour la médecine ambulatoire en Suisse

En date du 28 avril 2016, la Chambre médicale de la FMH a demandé une votation générale afin de recueillir l'avis des membres ordinaires de la FMH sur la révision du tarif ambulatoire. Vous êtes concerné et avez donc la liberté de donner votre avis au sujet du nouveau tarif. Il vous appartient dès lors de faire parvenir votre bulletin de vote au notariat dont l'adresse est libellée sur l'enveloppe, d'ici au 28 mai 2016.

Durant ces derniers quatre ans, environ 200 délégués tarifaires des sociétés de discipline médicale de la FMH, de concert avec les experts tarifaires des partenaires de la révision $\mathrm{H}+$ et la CTM, se sont attelés au remaniement complet du tarif médical ambulatoire. $\mathrm{Au}$ prix d'un nombre incalculable de séances, ils ont ensemble révisé plus de 4500 positions. A la fin mars

En tant que médecin, vous devez pouvoir facturer les prestations que vous avez effectuées.

2016, nous avons mis à votre disposition la structure remaniée, à laquelle vous pouvez désormais accéder via la plate-forme myFMH. Le 28 avril 2016, la Chambre médicale a décidé de consulter tous les membres ordinaires de la FMH lors d'une votation générale sur le tarif médical ambulatoire.

\section{Mise à jour du calcul des unités fonctionnelles}

Bien que datant déjà de plusieurs années au moment de l'introduction du TARMED en 2004, les bases de calcul continuent d'être utilisées aujourd'hui encore, sans pour autant avoir subi de modifications. Il est toutefois indéniable que la situation dans le domaine des coûts s'est dans l'intervalle largement modifiée. Tandis que les coûts d'utilisation, principalement des appareils et installations, ont diminué, les frais de personnel ont nettement augmenté. Des estimations de la Caisse des médecins et de la FMH, fondées sur les chiffres de l'enquête permanente RoKo, font état d'une augmentation des coûts globaux de presque $30 \%$ ces dernières années. L'un des buts primordiaux de la révi- sion était donc de recalculer ces coûts, puis de les prendre en compte lors de la mise à jour des prestations.

Pour ce faire, les experts tarifaires ont alimenté avec des données actuelles les trois modèles de coûts à prendre en considération, soit la «Prestation médicale», «KoReg» et «INFRA». Pour chacune des prestations, ils ont calculé la valeur du point de la prestation médicale (PM) et celle de la prestation d'infrastructure et de personnel (PIP, anciennement prestation technique PT). Nous avons eu recours au principe dit d'opportunité pour déterminer le revenu de référence, paramètre le plus important de la prestation médicale. On admet dans ce cadre que, durant toute la durée de sa vie active, le travail d'un médecin en pratique privée génère un revenu correspondant à celui d'un médecin hospitalier au bénéfice d'un contrat de durée indéterminée. On divise ensuite cette valeur de référence par le temps de travail annuel exprimé en minutes. Ce temps de travail annuel englobe non seulement les week-ends et jours fériés, mais aussi les absences dues par exemple à des vacances, une grossesse, une maladie ou la formation. Dans le cadre du modèle INFRA,

\section{Urs Stoffel au sujet des alternatives}

\section{à la révision tarifaire}

Pour Urs Stoffel, membre du Comité central de la FMH et responsable du département Tarifs et conventions pour la médecine ambulatoire en Suisse, il est évident qu'il n'existe pas beaucoup d'alternatives: "Lors de plusieurs prises de position et entretiens, le Conseil fédéral a toujours signalé qu'il exigeait de la part des partenaires tarifaires une proposition pour le 30 juin 2016, faute de quoi il interviendra à nouveau au niveau du tarif.» 
Révision du tarif médical ambulatoire Documentation

Vous trouverez des informations détaillées relatives au tarif médical révisé sur la plate-forme myFMH à l'adresse suivante: https://myfmh.fmh.ch $\rightarrow$ Révision tarif ambulatoire $\rightarrow$ Documentation.

l'ensemble des appareils et installations ainsi que tous les paramètres relatifs aux frais de personnel ont été actualisés en vue de déterminer les taux des charges PIP. Nous avons aussi alimenté le modèle KoReg avec des valeurs actuelles de l'enquête permanente RoKo afin de prendre en compte le développement des coûts durant ces dernières années.

\section{Révision de la nomenclature}

Une révision des modèles de coûts s'accompagne nécessairement d'une adaptation de la nomenclature correspondante. Le désaccord entre les partenaires tarifaires ayant bloqué ces dernières années une révision de la structure tarifaire, il y a maintenant, dans ce domaine aussi, un besoin considérable de rattrapage. Il existe aujourd'hui des prestations qui ne peuvent être facturées aux assureurs que par le biais de positions analogiques ou, inversement, des prestations qui, compte tenu des progrès de la médecine, ne sont plus utilisées.

Malgré la tarification de nouvelles prestations dans de nombreux domaines, il a été possible de réduire à 2700 le nombre de positions - plus de 4500 aujourd'hui. De plus, la mécanique et les règles tarifaires ont été foncièrement simplifiées. Du point de vue des partenaires de la révision, les limitations actuellement en vigueur ne revêtent plus de sens sur le plan médical ni ne protègent des abus tarifaires. Elles ont donc été abandonnées. Les partenaires ont aussi simplifié les interdictions de cumul: en tant que médecin, vous devez pouvoir facturer les prestations que vous avez effectuées. Aux yeux des partenaires de la révision, il reste indéniable que toute prestation doit être efficace, appropriée et économique au sens de l'art. 32 al. 1 de la LAMal.

\section{Transcodage et normalisation}

En août 2014 déjà, l'Office fédéral de la santé publique (OFSP) a informé les partenaires que le Conseil fédéral n'entérinera qu'une révision du tarif respectant la neutralité des coûts (OAMal art. 59c). L'OFSP écrivait alors: «S'il est prescrit, eu égard au principe d'économicité prôné par la LAMal, qu’à données semblables un changement de modèle ne doit pas entraîner de coûts sup- plémentaires, il y a lieu d'aussi appliquer ce principe lorsqu'une révision tarifaire va moins loin qu'un changement fondamental de modèle tarifaire.» Outre cette neutralité des coûts plutôt statique, l'OFSP exige clairement une neutralité des coûts dite dynamique. L'OFSP demande en effet aux partenaires tarifaires de présenter sous forme de concept les conséquences et mesures à envisager si, dans le futur, la nouvelle structure tarifaire devait occasionner une augmentation du volume global des prestations. Les exigences des assureurs vont dans la même direction.

Il faut en effet prendre en considération l'évolution réelle des coûts lors des négociations relatives à la valeur du point tarifaire.

Compte tenu de l'augmentation du volume des points tarifaires, la FMH accepte une neutralité des coûts statique sur le plan de la structure, c'est-à-dire pour le volume des points tarifaires au moment de l'introduction de la structure, mais non sur le plan du prix. Il faut en effet prendre en considération l'évolution réelle des coûts lors des négociations relatives à la valeur du point tarifaire. C'est la raison pour laquelle les partenaires ont décidé conjointement d'une normalisation de la structure tarifaire remaniée.

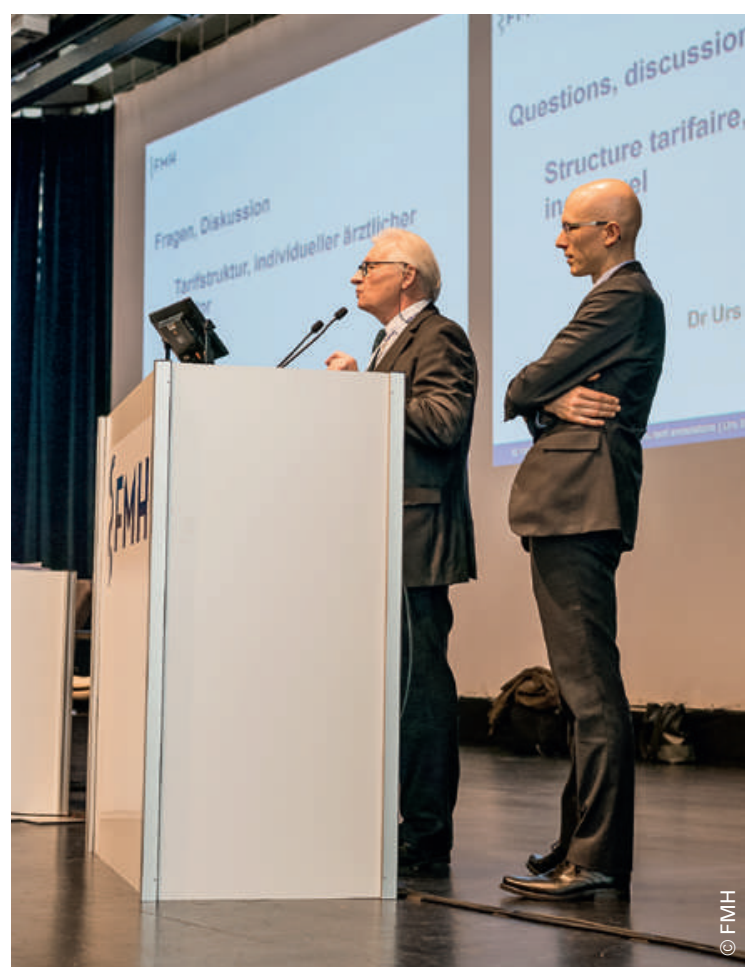

Urs Stoffel du Comité central de la FMH et Patrick Müller, chef de la division Tarifs et conventions pour la médecine ambulatoire en Suisse, répondent aux questions des délégués à la Chambre médicale. 


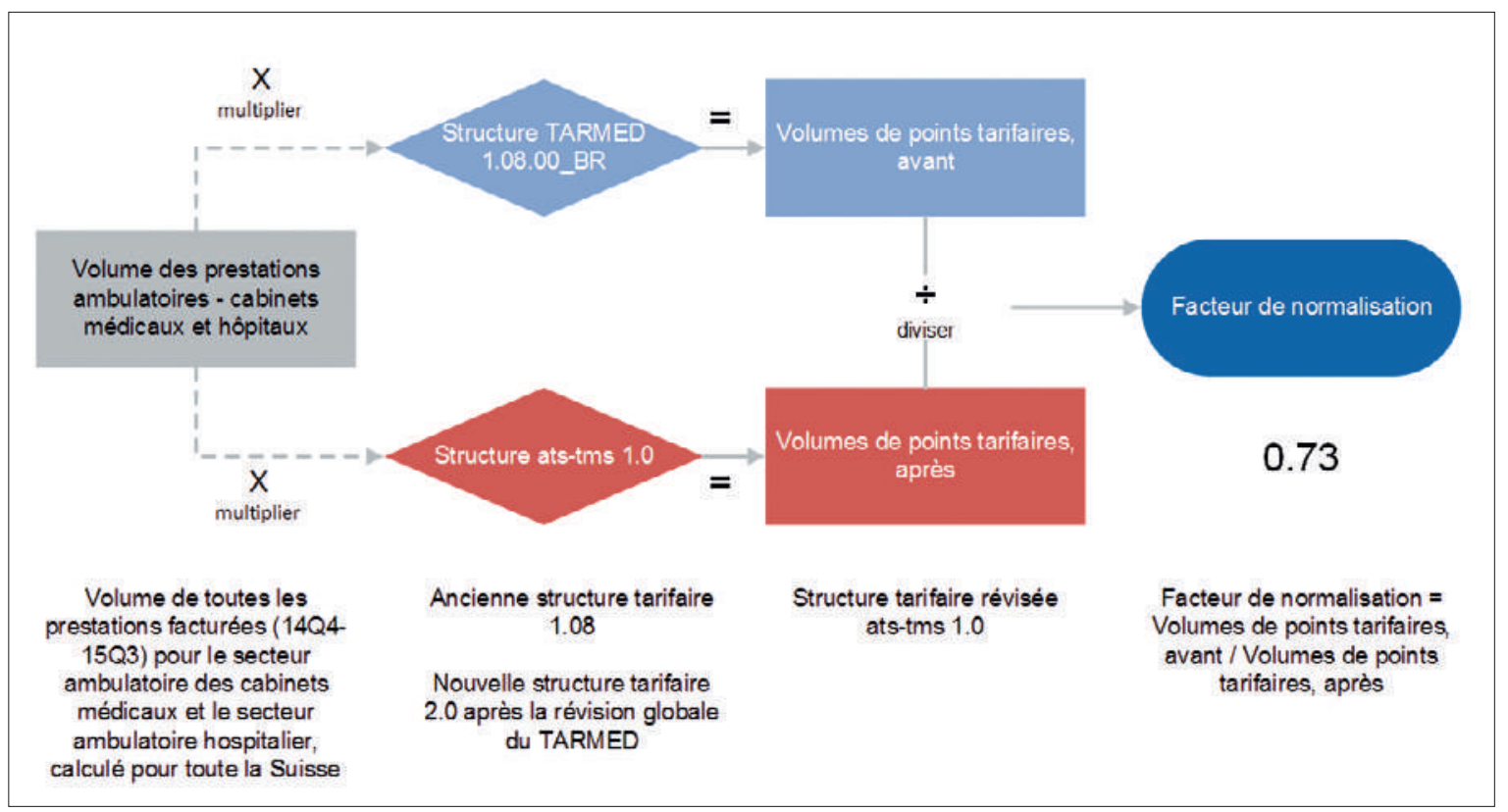

Figure 1: Une augmentation des coûts de $37 \%$ serait enregistrée sans la normalisation de la structure tarifaire. (IIlustration mise à disposition par l'auteur)

\section{Bilan intermédiaire: qu'avons-nous atteint?}

Vos collègues ont atteint plusieurs objectifs! La simplification de la structure devrait à l'avenir en améliorer la vue générale et, après une période d'adaptation, la mise en pratique. La facturation de toutes les presta-

Dans la structure tarifaire révisée, le temps de changement est pris en compte sous forme de positions tarifaires séparées.

tions effectuées en ambulatoire étant maintenant possible, il ne sera plus nécessaire de recourir à la facturation par analogie. Les prestations effectuées, calculées et mises à jour en fonction de critères d'économie d'entreprise, sont le reflet de la situation actuelle. La nouvelle structure organisationnelle d'ats-tms SA permettra en outre de réagir à l'avenir avec plus de célérité en cas de modification des conditions-cadres.

Il y a cependant quelques nouveautés. Il vous appartient, en tant que médecin, d'en tenir compte avant de facturer les prestations que vous avez effectuées.

\section{Modifications d'ordre général dans le nouveau tarif}

Afin d'éviter toute confusion entre le tarif TARMED actuel 1.08.00_BR et le tarif ats-tms 1.0, les positions tarifaires individuelles ont passé d'une désignation purement numérique à une numérotation alphanumérique.

\section{Utilisation du tarif à l'aide d'exemples concrets}

Prestations de base médicales et non médicales De nombreuses réactions, émanant autant de sociétés de discipline médicale que des assureurs, ont amené les partenaires de la révision à revoir une nouvelle fois le chapitre des prestations médicales de base, dont la simplification initiale avait été trop rigoureuse. Le point majeur de la critique portait essentiellement sur la concentration trop radicale du contenu, qui ne permettrait pas de suffisamment différencier les médecins et les spécialités en cas d'éventuelles procédures EAE.

La proposition finale permet de distinguer d'une part entre consultation, visite et consilium, d'autre part entre rapport médical et prestation médicale de base sans contact direct avec le patient. Des positions spécialisées relatives au traitement ou à des conseils ont à nouveau été créées dans de nombreux domaines, chacune d'entre elles étant basée sur la même évaluation (mêmes barèmes de coûts par minute), tout en permettant de différencier les prestations médicales de base. Il existe ainsi un nouveau chapitre «Traitements et conseils médicaux spécialisés» regroupant des prestations spécifiques pour les médecins au béné- 


\section{Urs Stoffel au sujet de la normalisation}

Une expertise juridique mandatée conjointement par la FMH et la Caisse des médecins dans le cadre du projet Concerto démontre que, conformément à l'art. 59c al. 1c OAMal, une normalisation du tarif révisé sur la base des volumes de points tarifaires de l'ancien tarif ne peut avoir lieu que lors d'un changement de modèle. De I'avis de la FMH, la normalisation du tarif médical révisé exigée par l'article 59c remet en question l'économicité et l'adéquation prônées par la LAMal, car ces dernières sont dans une large mesure altérées par le facteur de normalisation.

Lors des négociations du prix dans chaque canton, il faudra assurément prendre en considération les prescriptions de la LAMal relatives à l'adéquation et l'économicité d'un tarif.

fice de titres de spécialiste, de formations approfondies ou d'attestations de formation complémentaire, qui jusqu'à présent ne figuraient dans aucun chapitre ou sous-chapitre dédié.

La création du nouveau chapitre consacré aux prestations de base non médicales constitue un changement essentiel. Ces prestations permettront de facturer à l'avenir des prestations non tarifées et séparément effectuées par le personnel. Les prestations non médicales ne sont cependant pas applicables aux prestations en rapport avec des prestations médicales généralement effectuées par du personnel non médical. Ces prestations sont aujourd'hui incluses dans la PIP de la prestation médicale correspondante.

Concrètement, cela signifie que les positions AM.0001 et AM.0002 sont toujours utilisées ensemble, la position AM.0001 correspondant à la seule prestation du

\section{Le nouveau terme "assistance médicale» remplace l'expression «assistance».}

personnel (l'assistante médicale en l'occurrence) et la position AM.0002 à la seule infrastructure. Il vous est ainsi possible par exemple de facturer une surveillance de trois heures (le patient occupe le local à disposition durant trois heures) durant laquelle l'assistante médicale, accomplissant d'autres tâches le reste du temps, ne se consacre au patient que durant dix minutes. Si, en tant que médecin, vous consacrez de votre temps à cette surveillance du patient, vous facturez en sus la position SK.0004 du chapitre AF.0O. La position AM.0003 est par contre réservée à des prestations non médicales effectuées dans une infrastructure simple, qu'il s'agisse par exemple de conseils, d'information et d'instruction au patient, de prises de sang ou de traitement de plaie.

\section{Temps de changement}

Le temps de changement est le temps moyen (minutage) pour la préparation (nettoyage, réorganisation, etc.) de l'infrastructure (unité fonctionnelle) entre le traitement de deux patients. Dans la structure tarifaire révisée, le temps de changement est pris en compte sous forme de positions tarifaires séparées. Il ne peut être facturé qu'une seule fois par unité fonctionnelle et par séance.

\section{Préparation et finition}

Le temps de préparation et de finition est le temps qui sert à préparer et à finaliser la prestation médicale en tant que telle (par ex. lavage stérile et habillement lors d'une intervention chirurgicale). Les temps de préparation et de finition des positions individuelles sont généralement pris en compte par une position tarifaire séparée. Le temps nécessaire à les effectuer, jusqu'à maintenant compris dans la position tarifaire concernée, doit nouvellement être facturé de façon séparée dans la plupart des chapitres.

\section{Le rapport comprend la documentation et} l'établissement de l'exposé des résultats.

\section{Rapport}

Le rapport comprend la documentation et l'établissement de l'exposé des résultats. En général, la fourniture de la prestation d'une part et la documentation ainsi que l'établissement de l'exposé des résultats d'autre part ont lieu dans des infrastructures différentes (unités fonctionnelles). Une infrastructure simple (salle de consultation) suffit souvent à documenter par écrit la prestation effectuée. Actuellement, l'indemnisation prévue à cet effet par le TARMED prend en compte une infrastructure partiellement trop bien dotée. C'est la raison pour laquelle a été définie une nouvelle approche pour la tarification et l'indemnisation du temps nécessaire à établir l'exposé des résultats. L'établissement de ce document est en principe à facturer par la nouvelle position AF.0004.

\section{Assistance médicale}

Le nouveau terme «assistance médicale» remplace l'expression «assistance». Sont reconnues comme assistance médicale les personnes ayant terminé leur formation de médecin et réussi leur examen final. Le doctorat ne représente pas ici une condition sine qua non, car les spécialistes ne doivent pas toujours le détenir. Les futurs médecins, donc les médecins en formation (cand. med.), font eux partie du personnel non médical, et non de l'assistance médicale. 
Le tariflimite à deux le nombre de personnes reconnues comme faisant partie de l'assistance médicale. La présence de ces personnes doit être nécessaire sur le plan médical. Elle doit aussi être prouvée et justifiée vis-àvis des assureurs. Pour autant qu'elle soit nécessaire sur le plan médical, l'assistance médicale peut en principe être étendue à toutes les positions. Elle doit toutefois être facturée de façon séparée, par le biais des positions SK.0001 et SK.0002.

\section{Innovations}

Les positions du chapitre ST, intitulé «Innovations», mentionnent seulement des positions nouvelles, non encore tarifées dans la structure actuellement en vigueur. L'utilisation de ces positions ne doit déboucher sur aucun cumul avec des prestations spécifiques tarifées à l'intérieur de la même unité fonctionnelle. Aux yeux des partenaires de la révision, ces positions représentent en quelque sorte un avertissement signalant que, dans un domaine déterminé, il y a eu passage du domaine stationnaire au domaine ambulatoire ou qu'une nouvelle méthode de traitement

La nouvelle tarification a conduit à de profondes modifications en ce qui concerne le déroulement opératoire et les processus d'anesthésie.

a vu le jour. La prise en compte spécifique de ces prestations dans le tarif doit être proposée à l'organisation tarifaire par la société de discipline médicale concernée dans les six mois suivant la première comptabilisation.

\section{Procédures opératoires et d'anesthésie}

La nouvelle tarification a conduit à de profondes modifications en ce qui concerne le déroulement opératoire et les processus d'anesthésie. Ainsi, par exemple, compte tenu de l'enchaînement des processus, les prestations individuelles ont été mieux différenciées les unes des autres, des prestations manquantes ont été identifiées puis intégrées, et des prestations contribuant à la sécurité du patient en phase opératoire ont été tarifées. Les prestations générales en phase opératoire sont regroupées dans le chapitre RQ de la nomenclature: «Prestations générales en salle d’opération».

\section{Perspectives}

Dans le but de faciliter votre appréciation et votre décision, la FMH a mis en ligne diverses simulations et comparaisons entre les deux structures tarifaires. Ces documents sont à votre disposition sur la plate-forme myFMH (https://myfmh.fmh.ch $\rightarrow$ Révision du tarif ambulatoire $\rightarrow$ Documentation). Examinez - sur la base de données concrètes provenant de votre cabinet médical ou de paniers de prestations établis par les sociétés de discipline - les répercussions du tarif sur votre situation, forgez-vous une opinion jusqu'au 28 mai 2016 et déposez alors votre bulletin de vote.

La FMH adresse ses vifs remerciements à toutes les personnes ayant participé à ce gigantesque projet pour leur soutien illimité et sans faille.

La FMH attend le résultat de la votation générale d'ici juin 2016. Pour autant que la majorité des membres de la FMH adhère au tarif médical proposé, la FMH soumettra ce dernier le 30 juin 2016 au Conseil fédéral pour approbation. Si le Conseil fédéral entérine le tarif, la FMH et les partenaires tarifaires en planifieront l'introduction pour le début 2018.

La structure et la philosophie du nouveau tarif ne sont pas nécessairement évidentes sur la seule base du navigateur. C'est pourquoi, en collaboration avec les partenaires et les sociétés de discipline concernées, la FMH planifie l'organisation de formations dès l'automne 2016.

\section{Un grand merci}

La FMH adresse ses vifs remerciements à toutes les personnes ayant participé à ce gigantesque projet pour leur soutien illimité et sans faille. Cette révision tarifaire n'aurait guère été pensable sans l'immense engagement des délégués tarifaires et des présidents des sociétés médicales. Beaucoup d'entre eux ont travaillé jour et nuit. Un grand merci à chacun! 